\title{
Identification of B52-dependent Gene Expression Signature and Altemative Splicing Using a D. melanogaster B52-null Mutant
}

\author{
Sunwoo Hong, ${ }^{a}$ Misun Jung, ${ }^{+, a}$ Eunkyung Kim, ${ }^{\dagger}$ Dong-ki Lee, and Soyoun Kim ${ }^{\dagger,}$, \\ Global Research Laboratory for RNAI Medicine. Department of Chemistry and BK2I School of Chemical Materials Science. \\ Sunghyunkwan Linversity, Sinwon 4+0-746, Korea. ${ }^{*}$ E-mail: dkleeaskkuedu \\ Department of Biomedical Engineering, Dongguk Unwersity, Seoul 100-715. Korea. E-mail: skimoidonggukedu \\ Received December 12, 2008, Accepted December 17, 2008
}

\begin{abstract}
SR proteins are essential splicing regulators and also nodulate altenative splicing events, which function both as redundant and substrate-specific mamer. The Drosophila B52/SRp55, a member of the SR protein family, is essential for the fly development in vivo, as deletion of B52 gene results in lethality of animals at the second instar larval stage. Identification of the splicing target genes of B52 thus should be crucial for the understanding of the specific developmental role of B52 in vivo. In this study, we performed whole-genome DNA microarray experiments with a B52-knock-out animal. Analysis of the microarray data not only provided the B52-dependent gene expression signature, but also revealed a larval-stage specific, altemative splicing target gene of B52. Our result thus provides a starting point to understand the essential function of B52 at the organismal level.
\end{abstract}

Key Words: B52/SRp55. DNA microarray. Alternative splicing

\section{Introduction}

Gene splicing in higher eukaryotes is regulated by a battery of protein factors that facilitates generation of appropriate splice variants. This spacial and temporal regulation not only increases the complexity of the proteome but is also required to achieve developmental stage and tissue specific gene expression. One of these regulatory protein factors involved in splicing are the SR proteins. which act as both essential splicing factors and alternative splicing regulators that function in the early stages of spliceosome assembly. ${ }^{\prime}$ SR proteins share a similar structure. with RNA recognition motifs (RRM) at the $N$-terminus and have a region rich in Arg-Ser dipeptides (RS domain) at the $\mathrm{C}$-terminus.

B52/SRp55. which was originally identified as a bracketing factor of heat shock puffs in polytene chromosomes, ${ }^{3}$ later turned out to be a member of the SR protein family. B52 is an essential splicing factor in Drosophila melanogaster both in vitro and in vivo. Further, it has also been shown to be essential for ftz splicing in splicing-deficient $\$ 100$ extract in vitro. ${ }^{3}$ Also. B52 deficiency in animals is lethal at the second instar larval stage. suggesting the essential. non-redundant function of $\mathrm{B} 52$ in vivo. ${ }^{4}$ Targeted disruption of other SR proteins such as $\mathrm{ASF} / \mathrm{SF} 2^{5}$ and $\mathrm{SC} 35^{6}$ in mice also supports the idea of non-overlapping role of SR proteins in vivo.

Recently studies in this field have uncovered the specific alternative splicing events regulated by different splicing regulators. Blanchette et al. combined RNA interference (RNAi) and DNA microarray techniques to identify' splicing target genes of several splicing regulators including B52 in Drosophila SL2 cells. Another study also utilized RNAi and microarray to identify 11 target genes of huRNP L ${ }^{8}$ Recently, splice site-specific DNA microarray analysis of human U2OS cells treated with siRNA targeting \$Rp55 revealed KSR1. ZAK. and mda $7 / / \mathrm{L} 24$ as splicing target genes. ${ }^{9}$ While these

\footnotetext{
${ }^{a}$ These authors contributed equally.
}

studies are informative in providing a comprehensive list of potential splicing target genes. alternative splicing observed in cell lines might not reflect the ply siological importance of splicing regulators at the organismal level. For example. B52 is the predominant splicing factor in Drosophila $\mathrm{Kc}$ cells. but not in whole embryos. ${ }^{3}$

In wivo studies. mostly carried out in flies. have identified several in vivo splicing target genes of SR proteins. B52 was identified as a splicing regulator of $\mathrm{dE} 2 \mathrm{~F} 2$ in flies. ${ }^{10}$ Overexpression studies identified several altemative splicing target genes of B52 and ASF/SF2 in developing fly eye, and a recent study ${ }^{12}$ showed that the alternative splicing of eyeless gene is specifically regulated by B52. suggesting that B52 might play a critical role in normal eye development in flies. While these studies have been performed in specific tissue types, they clearly demonstrate the specific role of splicing factors in vivo.

To gain an insight into the essential role of B52 in fly embryonic development. we have been studying the B52 knock-out flies. ${ }^{+}$Using genonic SELEX technology, we have previously identified several genes whose altenative splicing pattern was changed in B52-null mutant flies. The expression levels of B52-target genes identified were sinular in both the B52 wild-type and mutant animals. In this study. to test whether B52 depletion affects the global gene expression signature. we explored the gene expression changes triggered by the depletion of B 52 in fly embryos by using the whole genome DNA microarray. From this experiment, we were able to identify a number of genes whose expression level is affected by B 52 depletion. We also report the finding of a novel gene whose alternative splicing is regulated by B52 during the larval development

\section{Experimental Section}

Fly preparations. B52 depleted mutant flies were described previously. ${ }^{4}$ In brief, homozy gous $\mathrm{B} 52$ mutants $\left(B 52^{-8}: B 52^{-18}\right)$ 
were picked 7 days after laying eggs. Homozyous B52 larvae were distinguished from heterogeneous B 52 wild type by non-tubby and tubby shapes respectively: About 150 non-tubby and 10 tubby larvae were hand-picked and frozen with TRI reagent (SIGMA. USA) in $-70^{\circ} \mathrm{C}$. Secondary- and third-instar larvae of Oregon $R$ were used to test for the larval stage-dependent gene expression.

RNA isolations. Frozen larvae were homogenized in TRI reagent using Pellet Pestle' $\overline{\mathrm{B}}$ ) Motor (KONTES. USA) and disposable grinder. Total RNAs from larvae were punfied according to manufacturer's protocol and treated by DNase I to remove genomic DNAs. The concentration of total RNA prepared were determined using a NanoDrop ND-1000 spectrophotometer (NanoDrop Technologies. USA) and RNA integnty was verified by $1 \%$ agarose gel electrophoresis. The RNA samples for microarray analysis were cleaned up using RNeasy Mini Kit (Qiagen. Germany) and stored at $-70^{\circ} \mathrm{C}$ before use.

Oligonucleotide micmarray, hybridization, image acquisition and data analysis. NimbleGen Drosophila melanogaster 1-Plex Array (Roche NimbleGen. USA) was used for gene expression profiling. Sample labeling and hybridization were done as described in mamufacturer's protocol. The prepared microarrays were scanned with an Axon GenePlx $4000 \mathrm{~B}$ scanner (Molecular Devices Corp. USA). The data were extracted from the raw images using NimbleScan software (Roche NimbleGen. USA). Relative signal intensities (log.) for each feature were generated using the Robust Multi-Array Average (RMA) algonthm.

Differentially-Expressed Genes (DEGs) selection. We converted the nomalized microarray data of each sample into the signal ratio (B52del/WT) which represents the relative expression level of a target transcript. A difference of more than 2xSD in the average of $\log 2$ (signial ratio) was considered to be accounting for the up-regulated genes in B52 mutants. while a difference of less than 2XSD was defined as representing a down-regulated gene in B52 mutants. Among the 15.634 fnit fly transcripts present on the NimbleGen oligonucleotide array. $13.3 \%$ changed their expression in both samples.

Gene Ontology analysis. GO analysis was performed through Database for Annotation, Visualization. and Integrated Discovery (DAVID) platform. Selected DEGs list was uploaded onto DAVID website (http//niaid.abcc.nciforf.gov/ home.jsp) and using the total genes of Drosophila melongaster as background. enrichment of specific GO term was identified.

cDNA synthesis and PCR reactions. $I \mu \mathrm{g}$ of total RNAs per $20 \mu$ reaction were reverse-transcribed with ThemoScript Reverse Transcriptase (Invitrogen. USA). RT reactions were done $\mathrm{m} 50^{\circ} \mathrm{C}$ for 2 hours and treated with RNase $\mathrm{H}$ (Invitrogen) at $37^{\circ} \mathrm{C}$ for 30 minutes. $1 \mu$ of cDNA was used as a template for each PCR. PCR (in $25 \mu \mathrm{l}$ ) was performed as follows: 30 seconds at $95^{\circ} \mathrm{C}$. 30 seconds at $58^{\circ} \mathrm{C}, 30$ seconds at $72^{\circ} \mathrm{C}$ for 35 cycles. followed by $72^{\circ} \mathrm{C}$ for 7 munutes. The PCR was performed using primer sets as shown in Supplementary Table 1.

\section{Results and Discussion}

Genome-wide gene expression analysis of B52 deletion mutant. To understand the comprehensive gene expression profile in B52 deletion nutant animals. we performed a genome-wide DNA microarray expenment. Total RNAs from B52 mutant and wild type were extracted and the microarray experiment was conducted by using the NimbleGen Drosophila melanogaster 1-Plex Array platform. which contains multiple probes of about 15,000 Drosophila transcripts. We obtained the expression ratio of each gene and extracted differentially expressed genes (DEGs) in B52 deletion mutant flies. For the selection of DEGs. we calculated standard deviation of gene expression ratio and defined genes which showed higher or lower gene expression ratio than 2 S.D. as DEG. As a result. we identified 278 up-regulated and 854 down-regulated genes in B52 deletion mutant flies.

To characterize these DEGs. we performed gene ontology analysis. First we analyzed up-regulated genes and found that only two terms were significantly enriched ("polysaccharide metabolic process' and 'chitin metabolic process', $p<0.001)$. For down-regulated genes. we found that diverse gene ontology terms were enriched (Fig. 1). For biological process category, ontology analysis reported signuficantly enriched terms such as 'spermatogenesis". 'microtubule-based process'. and 'ATP biosynthetic process". We also found that cellular component analysis showed enrichment of 'microtubule cytoskeleton' and 'mitochondrion'. These results suggest that the impairnent of B52 function affects expression of genes engaged in specific cell lineage differentiation and inner cellular stnucture organization. In particular. we found reduced expression of genes which function in crucial processes for sustaining cell viability. For example. some downregulated genes were found

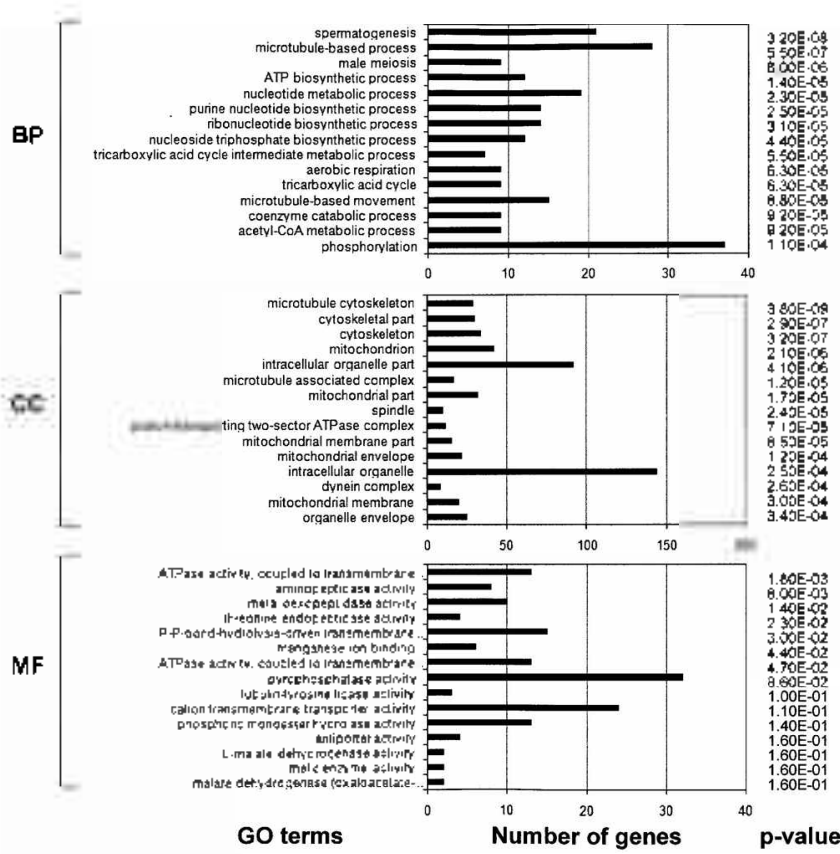

Figure 1. Gene ontology analysis of down regulated genes in B52 deletion mutant. List of 854 down regulated genes in B52 deletion mutant analyzed by web-based gene ontology tool. Enriched GO terms were sorted by reported p-values and 15 most enriched terms of three categories |Biological process (top panel i, Cellular component (middle panel), and Molecular function (bottom panel)| are presented. 
to be involved in synthetic process of ATP which is a unversally important coenzyme and enzyme regulator. In addition, genes involved in tricarboxylic acid cycle were also found as B52-dependent genes. These results indicate that the impairment of B52 causes deleterious effects on the organism and may explain the lethality of $\mathrm{B} 52$ deletion in anmal development.

B52 depletion affects altemative splicing of CG32548 gene at the organismal level. Multiple probes for individual gene in NimbleGen array format allowed us to examine the differential expression of individual exons within a specific gene. Each probe set of NimbleGene Drosophila expression mic-

(A) Gene Structure

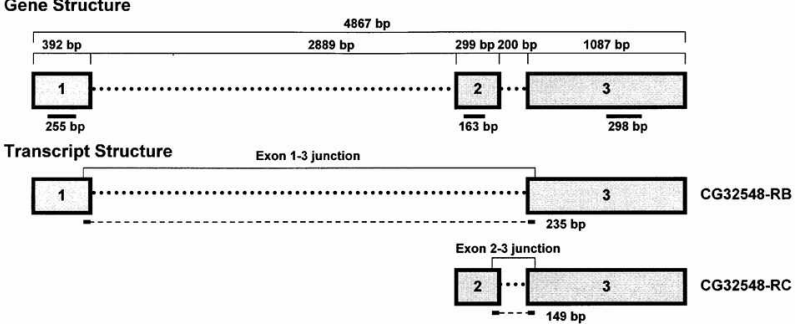

(B)
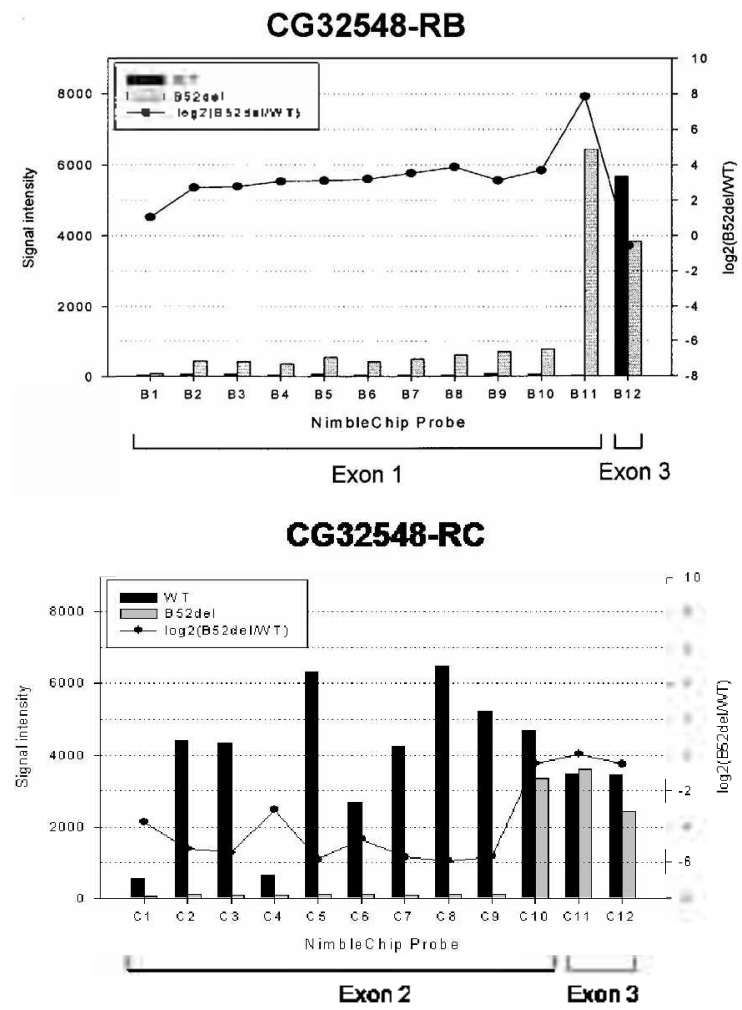

Figure 2. Expression level of CG32548 in micnoarmy analysis and primer design for RT-PCR validation (A) Primer design for CG32548. Target site and PCR product of each exon-specific primer set are represented on the gene structure (upper panel). Target site and PCR product of each transcript variant-specific primer set are indicated on the transcript structure (lower panel). The same reverse primer was used for the RT-PCR anal sis of C $\mathrm{G} 32548-\mathrm{RB}$ and RC. (B) Espression level of two CG 32348 transcript variants in Ninble Gen expression microarray analysis. Expression level of each transcipt variant is represented by the signal intensity (left Y-axis) of each probe for target transcript variant. Fold change of each transcript expression is indicated by the signal ratiollog2(B52del/WT)l (right Y-axis). Target exon of each probe is shown undemeath the graph. roarray is composed of 12 different oligonucleotide probes designed for targeting a single transcript. Some of these probes are designed for targeting common exons and the others for transcript-specific exons. We inspected the array data from B52 mull mutants and found that. the expression of transcript variauts of a gene named CG32548. was differentially regulated in B52 null mutants. CG32548 gene has two alternatively spliced transcript variants (Fig. 2A). Microarray data revealed that CG32548-RB transcript. which consists of exon 1 and exon 3, was up-regulated (Fig. 2B). In contrast. CG32548-RC. another transcript variant which consists of exon 2 and exon 3. was down-regulated in B52 null mutants (F1g. 2B). CG32548 was previously identified as one of the conserved polyglutanine proteins in Drosophila species with unknown function ${ }^{13}$

To validate the microarray data. we designed the pnimer sets specific for each exon and transcript vanant as shown in Figure 2A. RT-PCR analysis was performed to measure the expression of each CG32548 exon (Fig. 3. panels Exon 1, Exon 2 and Exon 3) in wild-type (Fig.3. lane 1) and B52-null mutant (Fig.3, lane 2). As shown in Fig. 3, RT-PCR data confirmed the expression patterns of each exon from microarray analysis. Whereas the expression of exon 3 , a common exon of CG32548, was not significantly changed. the expression of CG32548-RB specific exon I was up-regulated and that of CG32548-RC specific exon 2 was down-regulated by the loss of B52

As previously observed the homozy gous B52-null mutants used for this study showed growth arrest and died in $2^{\text {nd }}$ instar

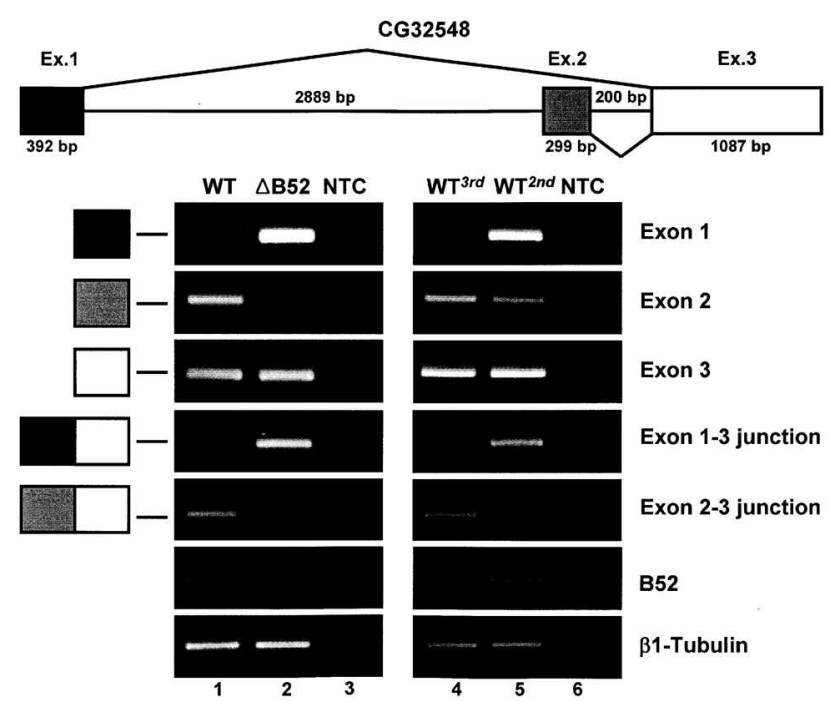

Figure 3. Changes in expression of CG32548 transcript variants under the loss of B52 and during the lar al life of D. melanogaster. RT-PCR of CG32548 was performed with primer sets specific for each exon or transcript variant as shown in Fig. $2 \mathrm{~A}$. The genomic organization and splicing scheme of CG 32548 gene are slummariced in the top panel. Three exons are represented by black, gray, and white boxes, respectively, and targets of each RT-PCR are also represented by these exon-specific boxes in the left panel. To test the effect of $B 52$ deletion in CG32548 expression, wild-type (tubby $3^{\text {th }}$ instar larvae of $\mathrm{B} 52^{28} / \mathrm{B}_{5} 2^{28}$. WT. lane 1 ) and $\mathrm{B} 52$-null mutant (non-tubby $2^{\text {id }}$ instar larvae of $\mathrm{B} 52^{28} / \mathrm{B} 52^{28}, \Delta \mathrm{B} 52$, lane 2) were used. $3^{\text {id }}$ instar larvale (WT ${ }^{3 r a}$. lane 4 ) and $2^{\text {ind }}$ instar larvale (WT ${ }^{3 n k i}$. lane 5 ) of Oregon R were used to analyze the patten of CG32548 expression during the larval stage. Bl-Tubulin was used as an intemal control and NTC (No Template Control. lane 3 and 6) was ulsed as a negative control 
larval stage. ${ }^{4}$ To confirm whether the expression patterns of CG32548 transcript variants was caused by the lack of B52 splicing activity or by lanal growth arrest. we performed RT-PCR analysis on total RNA from the wild-type larvae (Oregon R) collected in two different larval stages. CG32548$\mathrm{RB}$ was up-regulated in $2^{\text {nd }}$ instar larvae. and then downregulated in $3^{\text {td }}$ instar larvae (Fig. 3, panel Exon 1-3 junction. lanes 4 5). Considering that CG32548-RB was also upregulated at the $2^{\text {tw }}$ instar larval stage in the B52-null mutant although the mutant cannot survive beyond this stage. these results suggest that the loss of B52 affected the decrease of CG32548-RB transcript level during the transition stage between $2^{\text {nd }}$ and $3^{\text {rd }}$ instar larval stage. In contrast, CG32548$\mathrm{RC}$ transcript. which was down-regulated in B52 null mutant. was consistently expressed during the larval life of wild-type animals (Fig. 3. panel Exon 2-3 junction lanes 4 and 5). This result clearly demonstrates that the loss of B52 affects the exon 2 usage of CG32548 gene during the Drosophila larval development. and thus reduces the expression of CG32548$\mathrm{RC}$ transcript variant in $3^{\text {td }}$ instar larval stage.

In this work. we reported that the expression of CG32548 transcript variants during Drosophila larval development. are different in B52 null mutant as compared to that of the wild-type larvae. In line with our previous report. ${ }^{3}$ the current study provides additional evidences supporting the idea that $\mathrm{B} 52$ regulates the alternative splicing of genes during the larval development. In the present study. CG32548-RB was expressed differentially during larval stages, while CG32548$\mathrm{RC}$ was expressed consistently. These results strongly suggest that the differential expression of CG32548 transcript variants is associated with Drosophila lanal development. Furthermore, the loss of B52 interfered with the decrease of CG32548$\mathrm{RB}$ expression during the transition between $2^{\text {nd }}$ and $3^{\text {rd }}$ instar larval stages and induced the decrease of CG32548-RC in $3^{\text {ril }}$ instar larval stage. Although further studies will be needed to verify the correlation between the amount of CG32548 transcript variants and their function during larval stages, our data brings it in focus as one of the main targets of $\mathrm{B} 52$ splicing mechanism. Additionally the B52 regulated temporal expression of CG2548 splice variants in Drosophila larval development is intriguing as B 52 deficiency has been reported to be lethal at the $2^{\text {ind }}$ instar larval stage.

Altogether, our data shows that loss of B 52 affects both the global gene expression pattern and alternative splicing. and thus explains the role of B52 in Drosophila developnental process.

Aclonowledgments. S. K. was supported by the National Research Laboratory Program (MOST). D.-k. L was supported by Global Research Laboratory program by KICOS (grant 2008-00582), the Korea Ministry of Environment and the POSTECH BSRI fund

Supporting Infomation. Supplementary Table I mentioned in the text is available at the bkcs website (htp ww kcsnet. or.kr.bkcs).

\section{Refeiences}

1. Kim, S.; Shi, H.; Lee, D. K.; Lis, T. T. Nikfeic Acids Res. 2003 , 31,1955 .

2. Champlin, D. T.: Lis, J. T. Mol. Biol. Cell 1994, 5, 71

3. Hoffinan B. E. Lis J. T. Mol Cell Biol 2000, 20,181

4. Ring, H. Z, Lis, J. T. Mol. Cell Biol. 1994, 14, 7499-506

5. Wang, J.: Takagaki, Y, Manley, T. L. Genes Dev. 1996, 10, 2588.

6. Xiao, R.: Sun, Y.: Ding. T. H.: Lin. S.: Rose. D. W.: Rosenfeld. M. G.; Fu, X. D.; Li, X. Mol. Cell Biol, 2007, 27,5393.

7. Blanchette, M. Green, R. E.; Brenter, S. E.; Rio, D. C. Genes Dev. $2005,19,1306$.

8. Hung, L. H.; Heiner, M.: Hui, I.: Schreiner, S.: Benes, V; Bindereif, A. R $12008,14,284$

9. Filippov, V: Schmidt, E. L; Filippova, M: Duerksen-Hughes, P. J. Gene $2008,420,34$

10. Rasheva, V. I.: Knight, D.; Bozko, P:; Marsh, K.; Frolov, M. V. Hol. Cell Biol. 2006, 26, 3468 .

11. Gabut, M.; Dejardin, J; Tazi, J.; Soret J. Mol. Cell Biol. 2007, 27.3087

12. Fic, W. Juge, F.: Soret, J.: Tázi, T. PLOS ONE 2007, 2, e253.

13. Bettencourt, B. R.; Hogari, C. C.; Nimali, M J. Biosci. 2007, $32,537$. 